\title{
Laser-induced Particle Removal from Silicon Wafers
}

\author{
P. Leiderer ${ }^{\mathrm{a}}$, J. Boneberg ${ }^{\mathrm{a}}$, V. Dobler ${ }^{\mathrm{a}}$, M. Mosbacher ${ }^{\mathrm{a}}$, H.-J. Münzer ${ }^{\mathrm{a}}$, N. Chaoui ${ }^{\mathrm{b}}$, J. Siegel $^{\mathrm{b}}$, J. Solis $^{\mathrm{b}}$, \\ C.N. Afonso ${ }^{b}$, T. Fourrier ${ }^{c}$, G. Schrems ${ }^{c}$, D. Bäuerle \\ a Department of Physics, University of Konstanz, Fach M676, 78457 Konstanz, Germany \\ ${ }^{\mathrm{b}}$ Instituto de Optica, CSIC, Serrano 121, 28006 Madrid, Spain \\ c Institut für Angewandte Physik, Johannes-Kepler-Universität Linz, 4040 Linz, Austria
}

\begin{abstract}
The cleaning of silicon surfaces from submicron dust particles has been studied by means of the "Steam Laser Cleaning" (SLC) process and compared to "Dry Laser Cleaning" (DLC) which is used nowadays in many applications. For SLC a thin liquid layer (e.g. a water-alcohol mixture) is condensed onto the substrate, and is subsequently evaporated by irradiating the surface with a short laser pulse. The DLC process, on the other hand, only relies on the laser pulse, without application of a vapor jet. We have systematically investigated the efficiency of these two processes for the removal of well-characterized polymer, silica and alumina particles of various sizes down to $60 \mathrm{~nm}$ in diameter, and have also studied the influence of light wavelength and laser pulse duration for nanosecond and picosecond pulses. The results demonstrate that for the gentle cleaning of silicon wafers SLC is a very efficient method and is superior to DLC. An effect which so far has only rarely been taken into account for laser cleaning is the field enhancement under the particles, which can give rise to serious surface damage, in particular when cleaning pulses in the picosecond and femtosecond range in the DLC are applied.
\end{abstract}

Keywords: Laser cleaning, particle removal, silicon wafers, field enhancement, surface damage

\section{INTRODUCTION}

The preparation of well-defined surfaces, extremely clean and free of dust particles, is a challenge of utmost importance for the rapidly growing field of nanotechnology [1]. With the typical dimensions shrinking to a hundred nanometers and below, any contaminant particle with comparable size can cause serious perturbations in the structuring process and will eventually lead to malfunction of a device. Of particular relevance in this context is silicon as the key material for micro- and nanoelectronics. Hence there is considerable interest in the proper cleaning of silicon wafer surfaces. Unfortunately, conventional methods such as ultrasonics are inefficient in removing particles with a size on the nanometer scale [2]. Therefore, new techniques have to be developed. One promising idea is the use of short laser pulses for particle removal, the so-called laser cleaning process [3-9].

Two concepts for removing small particles by means of laser cleaning have been pursued during the last years:

- The first one is "Dry Laser Cleaning" (DLC), where the surface to be cleaned is irradiated by a short laser pulse, and the cleaning action is supposed to rely on the the strong acceleration at the surface due to thermal expansion, which leads to inertia forces strong enough to overcome the forces responsible for particle adhesion.

- The second concept, "Steam Laser Cleaning" (SLC), makes use of a thin liquid film which is condensed onto the surface just before the laser pulse is fired. Again, the energy absorption in the substrate leads to a rapid temperature increase, but now also the liquid film is heated, and bubble nucleation at the solid/liquid interface and the subsequent explosive vaporization of the liquid cause the removal of contaminants. 
Here we report on a systematic study of both DLC and SLC, in which we have applied laser pulses with different wavelengths and with pulse durations ranging from nanoseconds down to the femtosecond regime, and have used a variety of particles as contaminants on commercial silicon wafer surfaces.

\section{EXPERIMENTAL}

\subsection{Sample preparation}

Most experiments dealing with the laser-induced detachment of particles from silicon surfaces have so far been carried out with materials like alumina powder, consisting of rather irregularly shaped particles. For a systematic investigation of the laser cleaning process it appears advantageous, however, to have particles with a well-defined geometry whose properties such as size or material can be chosen in a controlled way. For our investigations we therefore have used small monodisperse spheres of polystyrene (PS) [10] or silica [11], as they are available in colloidal suspensions. The particle diameters ranged from 60 to $990 \mathrm{~nm}$. All these different samples were prepared on the Si wafers in the same way: after rarefaction with isopropyl alcohol (IPA) the particle suspension was deposited on the wafer by a spin coating process. For samples with isolated particles, the coagulation due to capillary forces during the drying process had to be minimized. This was achieved by high evaporation rates of the colloidal suspension and a low concentration of the particles in it. In this way, we were able to prepare samples with mainly isolated spheres (>95\%, cf. Fig.1) at sufficiently high particle density so as that a good statistics of the cleaning process could be performed.

Fig. 1: A typical sample $(4.3 \mu \mathrm{m} \times 5 \mu \mathrm{m})$ before cleaning showing a random distribution of isolated colloidal PS spheres with a diameter of $480 \mathrm{~nm}$.

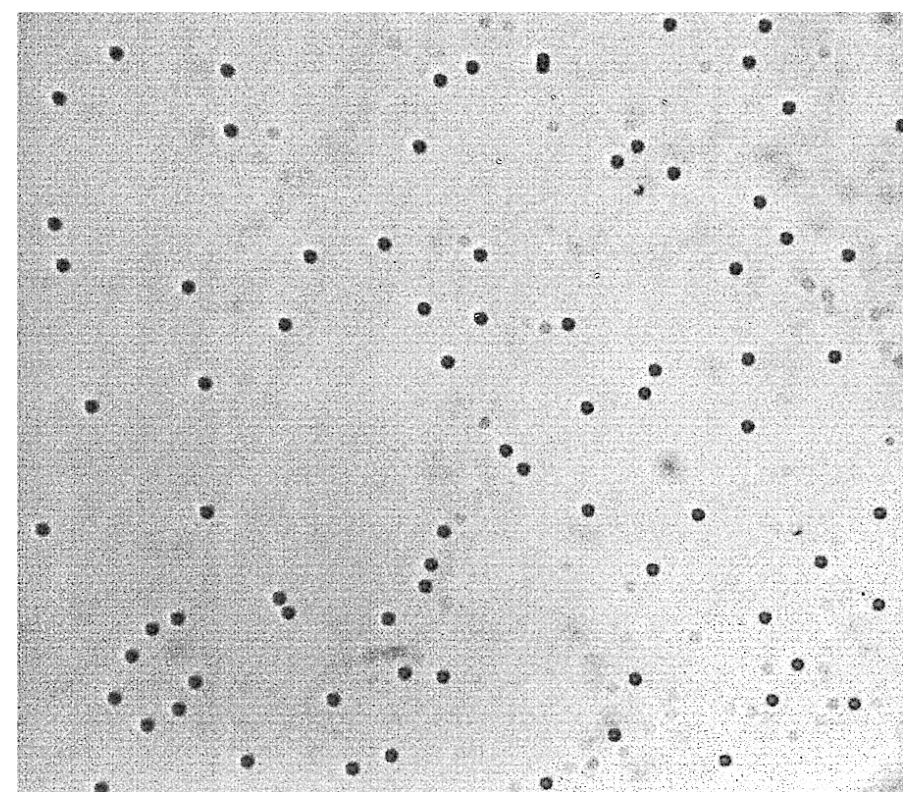

\subsection{Laser cleaning set-up}

The cleaning process was carried out using various laser systems: frequency-doubled Nd:YAG lasers $(\lambda=532 \mathrm{~nm}, \mathrm{FWHM}=$ $8 \mathrm{~ns}$ or $2.5 \mathrm{~ns}$ ), a pulsed dye amplifier seeded either with a cw dye laser beam or with a synchronously pumped mode-locked dye laser [12] $(\lambda=583 \mathrm{~nm}$, FWHM $2.5 \mathrm{~ns}$ or $30 \mathrm{ps})$, a mode-locked Ti:Sa oscillator/regenerative amplifier system $(\lambda=800$ $\mathrm{nm}$, FWHM from 150 fs to several ps), and a $\operatorname{KrF}$ excimer laser ( $\lambda=248 \mathrm{~nm}$, FWHM $29 \mathrm{~ns}$ ). An OPO pumped by a frequency-doubled Nd:YAG laser (FWHM=7ns) provided pulses at $\lambda=800 \mathrm{~nm}$ that enabled a comparison of the $\mathrm{ns}$ to the ps and fs regimes at the same wavelength.

The laser beams were focused to a spot size of about $1 \mathrm{~mm}^{2}$ at the sample site. The corresponding laser fluence incident on the sample was determined with several methods as described in detail in ref. [13]. For the SLC process, an additional burst of water/alcohol (10\% isopropanol) vapor was supplied through a nozzle above the wafer and condensed onto the substrate 
just before the laser cleaning pulse hit the surface [5]. The typical thickness of the liquid layer, as measured by ellipsometry, was about $300 \mathrm{~nm}$ just before the laser pulse was fired.

\subsection{Evaluation of the cleaning efficiency}

The efficiency of the particle removal process was either determined by simply counting the particles remaining on the surface using video microscopy, or - more conveniently - by measuring the particle concentration via light scattering [14]. For the latter method a 5-mW He-Ne laser was focused to the center of the cleaned region. The specular reflection of this probe laser beam was blocked and the scattered light was detected by a photodiode. Since the prepared samples consisted of mainly isolated particles that were not geometrically correlated, the scattered light intensity was to a good approximation proportional to the number $N$ of particles in the scattering area. The "cleaning efficiency", denoting the ratio of removed particles, is hence given by the quantity $\left(1-I / I_{0}\right)$, where $I_{0}$ and $I$ are the scattered intensities before and after the cleaning procedure, respectively. By direct comparison between light scattering measurements and optical microskopy we checked that the detection of the scattered light provides reliable quantitative information on the cleaning process [13].

The size of the region illuminated by the probe laser beam was in all cases of the order of $100 \mu$ m. This was much smaller than the cleaned area, thus it was ensured that a homogeneously irradiated region was monitored. At the same time, this spot was large enough to cover a sufficient number of particles at the concentrations used here to keep the statistical error small.

\section{RESULTS FOR STEAM LASER CLEANING}

\subsection{Steam Laser Cleaning with nanosecond pulses}

As already shown in previous work $[13,14]$ the cleaning efficiency for SLC with ns pulses $(\lambda=532 \mathrm{~nm}, \mathrm{FWHM}=8 \mathrm{~ns})$ exhibits a characteristic dependence on the applied laser fluence: Below a certain threshold value no particles are removed at all (i.e. the cleaning efficiency is zero), whereas above this threshold a steep increase of the cleaning efficiency is observed, until saturation to a value close to 1 is reached. This behavior is illustrated in Fig. 2 for PS spheres with diameters of $800 \mathrm{~nm}, 500 \mathrm{~nm}$, and $60 \mathrm{~nm}$. The data points were taken after 20 cleaning steps (i.e. condensation of liquid and subsequent laser shot) each. A photograph illustrating the efficiency for the $60 \mathrm{~nm}$ spheres, the smallest particles which to our knowledge have been removed by laser cleaning in a controlled way, is shown in Fig.3.

Fig. 2: Experimentally determined cleaning efficiency for various PS spheres vs. the applied laser fluence. The number of cleaning pulses from a Nd:YAG laser $(\lambda=532 \mathrm{~nm}, \mathrm{FWHM}=8 \mathrm{~ns})$ was 20 in each case. The cleaning threshold is sizeindependent, and the efficiency exhibits a steep increase above the onset of cleaning $\left(110 \mathrm{~mJ} / \mathrm{cm}^{2}\right)$. The same threshold is obtained when only 1 cleaning pulse is employed [14].

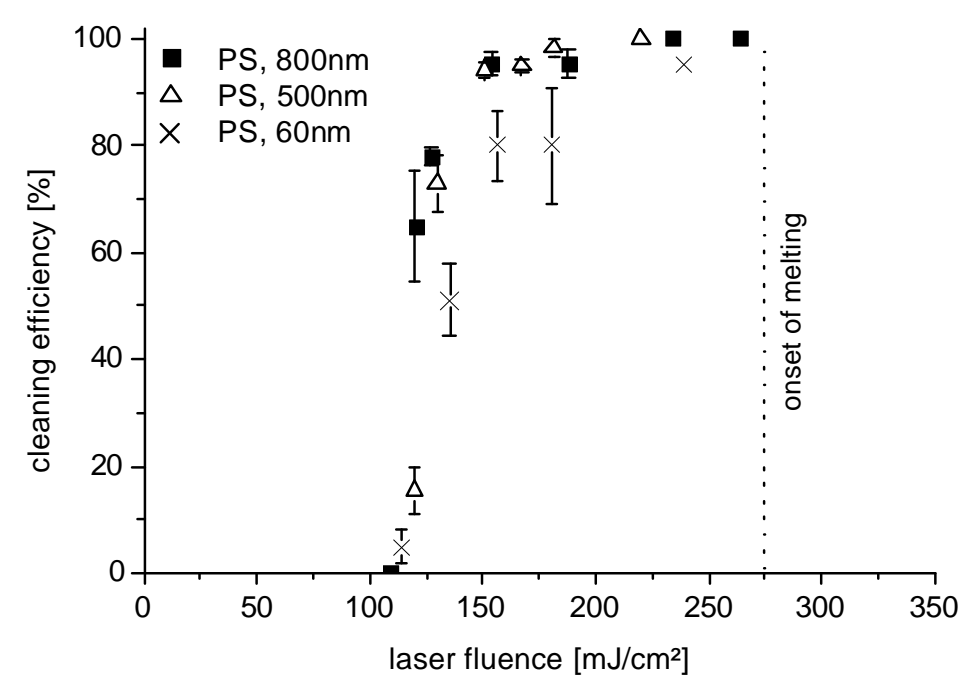


Fig. 3: Scanning Electron Microscope picture $(10 \mu \mathrm{m} x$ $6.5 \mu \mathrm{m})$ of a wafer surface contaminated with PS particles $60 \mathrm{~nm}$ in diameter. The left part, whose edge is marked by the white line, has been cleaned by ns SLC. The percentage of coagulated spheres was in this case realtively large, one can simultaneously judge the removal of individual spheres and of clusters.

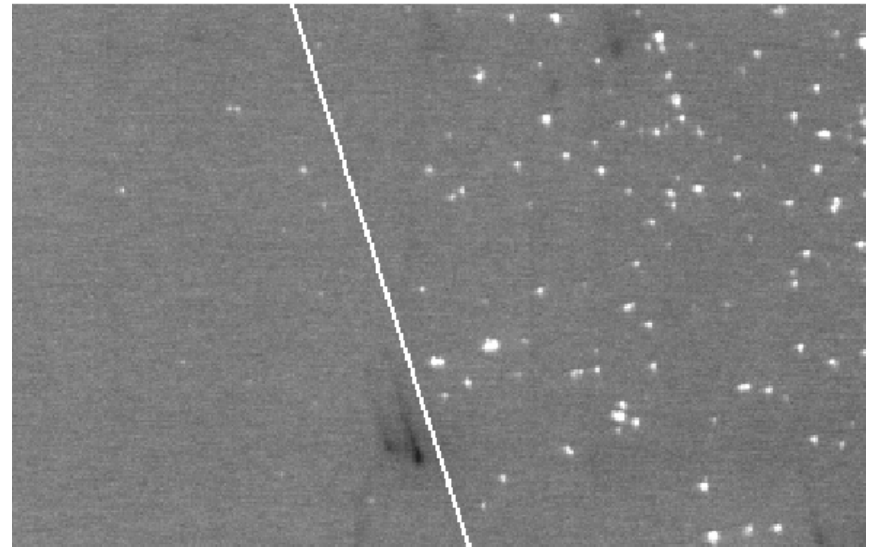

The onset for particle removal as plotted in Fig. 2 occurs at a laser fluence of $110 \mathrm{~mJ} / \mathrm{cm}^{2}$, irrespective of particle size. The same cleaning threshold was also found for PS spheres with diameters of $235 \mathrm{~nm}$ and $300 \mathrm{~nm}$ (not shown in the graph for the sake of clarity). At fluences above $170 \mathrm{~mJ} / \mathrm{cm}^{2}$ more than $90 \%$ of the particles are removed after 20 cleaning steps. For comparison, the threshold for melting of the bare Si surface has been marked in Fig. 2 and it is of $270 \mathrm{~mJ} / \mathrm{cm}^{2}$ for the used experimental conditions. The large separation between the thresholds for cleaning and melting is a considerable advantage with respect to industrial application where surface damage has to be strictly avoided.

In order to study the influence of the particle material on the cleaning efficiency we have also investigated silica spheres with diameters of $800 \mathrm{~nm}$ and $500 \mathrm{~nm}$ and (irregular) alumina particles with a mean diameter of $300 \mathrm{~nm}$ (Fig. 4). Once again we obtain the same dependence of the cleaning efficiency on the laser fluence and in particular the same threshold as for PS spheres. Experiments using slightly different laser parameters $(\lambda=583 \mathrm{~nm}, \mathrm{FWHM}=2.5 \mathrm{~ns}$ and $7 \mathrm{~ns}$ as well as $\lambda=532$ $\mathrm{nm}$ and FWHM=2.5 ns) showed again the same cleaning threshold [15].

Fig. 4: Experimentally determined cleaning efficiency for ns laser pulses $(\lambda=532 \mathrm{~nm}$, FWHM=8ns) and particles of different size, material and shape, plotted vs. the applied laser fluence.

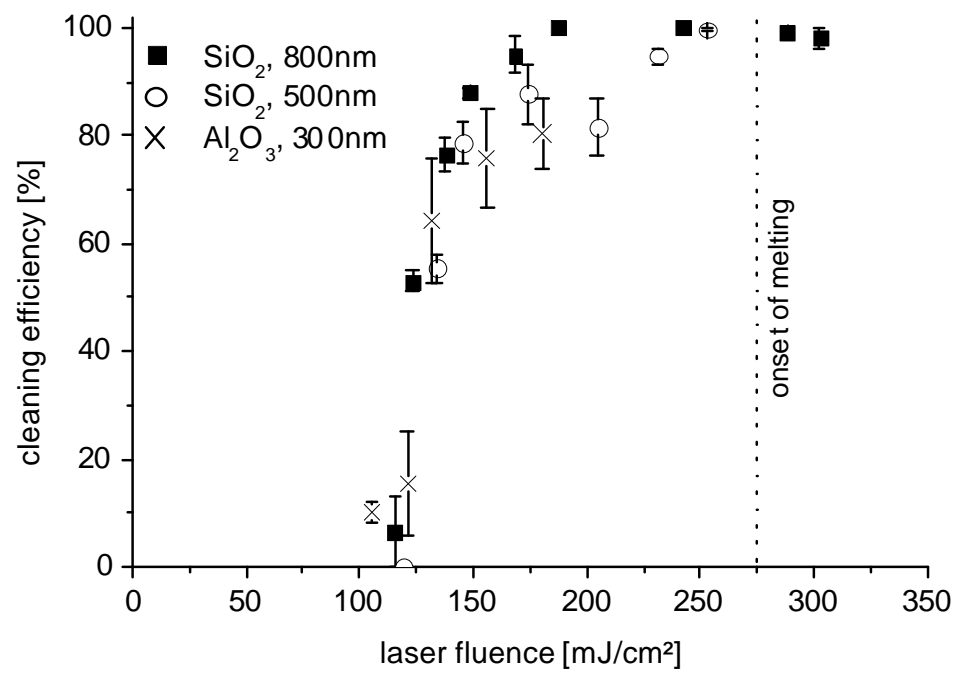

This quite universal behavior suggests that for the ns SLC process details of the particle-substrate interaction, which would depend on size, material and shape of the particles, are apparently irrelevant. The particle removal process is instead controlled by the explosive evaporation of the liquid film, whose onset is given by the nucleation of gas bubbles in the superheated liquid and should be insensitive to the parameters of the contaminant. It should be noted that for laser wavelengths in the UV a different threshold is to be expected, because the optical penetration depth in Si is much smaller in this regime compared to that in the $\lambda=532-583 \mathrm{~nm}$ range studied above and thus the heating of the interfacial region will be different; cf.Fig. 8. 
Fig. 5: Distribution of clusters of PS spheres, $800 \mathrm{~nm}$ in diameter, before (left) and after (right) the application of one cleaning pulse. The disintegration of the clusters due to the laser pulse is clearly visible.
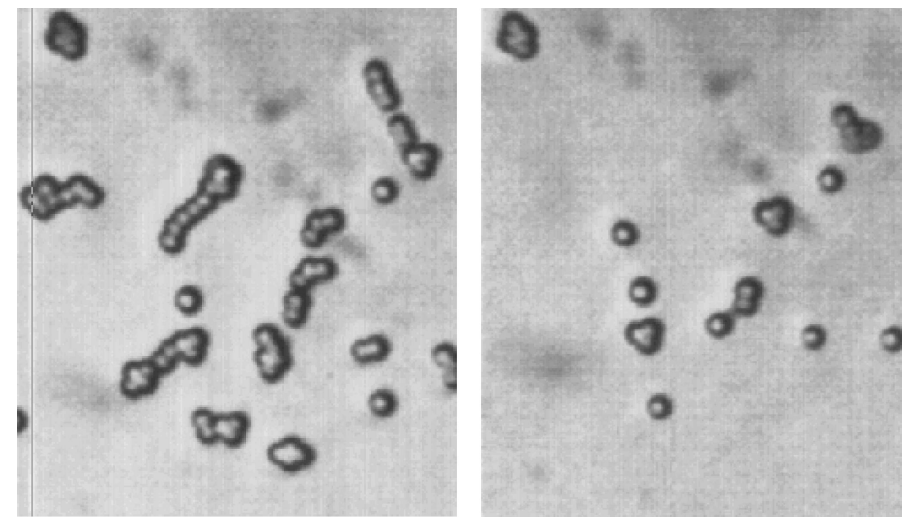

It has been reported recently that particle agglomerates are more easily removed by laser cleaning than individual particles [16]. In our experiments, we find the opposite behavior (see Fig. 5). Typically clusters of these regular particles are not removed as a whole, but are broken up step by step [14].

\subsection{Steam Laser Cleaning with picosecond pulses}

When the pulse duration is decreased from ns to ps, the amount of thermal energy diffusing into the Si wafer during the absorption of the laser pulse is reduced. This effect becomes obvious when one compares the thermal diffusion lengths in $\mathrm{Si}, 770 \mathrm{~nm}$ and $47 \mathrm{~nm}$ for an $8 \mathrm{~ns}$ and a $30 \mathrm{ps}$ laser pulse, respectively, with the optical penetration depth in Si (which is $1.7 \mathrm{um}$ at $583 \mathrm{~nm}$ ). The laser fluence required to heat the surface to a certain peak temperature will therefore be smaller for ps pulses as compared to ns pulses, which should have an influence also on the cleaning characteristics.

As an example for ps SLC we have plotted in Fig. 6 the cleaning efficiency as a function of laser fluence for pulses with $\mathrm{FWHM}=30 \mathrm{ps}$ and $\lambda=583 \mathrm{~nm}$. Since for the ps laser system the energy output fluctuations from pulse to pulse were much higher than those for the ns system, these fluctuations were monitored and recorded as the horizontal error bars in Fig. 6 . For comparison, the SLC cleaning threshold for ns pulses is included in the plot.

Fig. 6: Picosecond Steam Laser Cleaning efficiency $((\mathrm{FWHM}=30 \mathrm{ps}, \lambda=583 \mathrm{~nm})$ for $800 \mathrm{~nm}$ PS spheres. The melting threshold of silicon for these ps pulses as well as the cleaning threshold for ns SLC are indicated.

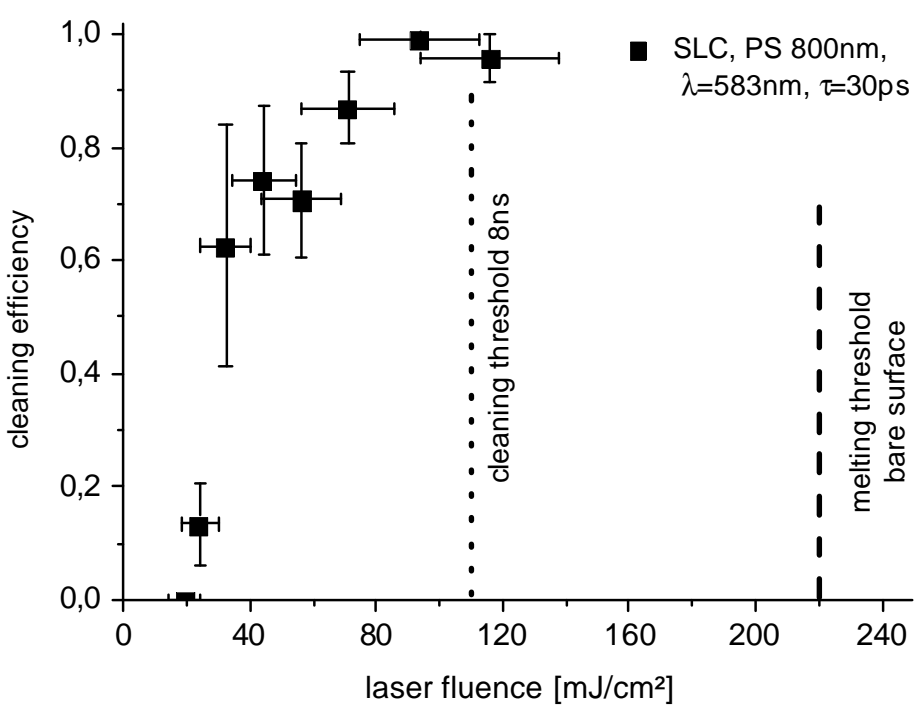


According to Fig. 6 a sharp cleaning threshold is also observed for ps pulses. However, the absolute value of this threshold is about $20 \mathrm{~mJ} / \mathrm{cm}^{2}( \pm 10 \%)$, a factor of 5 lower than for ns pulses. The fluence threshold for having a cleaning efficiency of $90 \%$ is also well below the corresponding value for ns SLC. This effect can qualitatively be ascribed to the reduced role of heat diffusion for the shorter pulses, and numerical simulations support this interpretation.

The data in Fig. 6 suggest that ps pulses might be even more suitable for SLC than ns pulses as the relative difference between cleaning threshold and melting threshold of the bare substrate surface is larger for the short pulses. One important aspect, however, has been neglected so far: Due to field enhancement underneath the particles the Si surface might locally melt even at average fluences well below the melting threshold. This is a much more serious problem for the shorter pulses, because, as already mentioned above, there the thermal energy cannot diffuse out of the heated region during the time of the pulse, giving rise to a much more pronounced local temperature increase than for longer pulses. As a result, the surface may be damaged locally, and indeed we found experimental evidance for this in DLC with ps and fs pulses as will be discussed below.

\section{RESULTS FOR DRY LASER CLEANING}

\subsection{Laser-induced particle removal}

As shown in the previous section, SLC is a very efficient method for removing submicron particles from wafer surfaces. The question that arises, though, is whether it is really necessary to apply a vapor jet just before the laser pulse, as described above, or whether essentially the same cleaning efficiency can be achieved under ambient conditions or even in vacuum. We have therefore carried out a series of measurements under conditions which are usually designated as "dry laser cleaning" (i.e. without additional liquid film).

Fig. 7: Cleaning efficiencies for ns Dry Laser Cleaning (40 laser shots each) and Steam Laser Cleaning (20 shots each). The DLC experiment was carried out in „normal“ laboratory atmosphere without the application of an additional vapor pulse as for the SLC experiment.

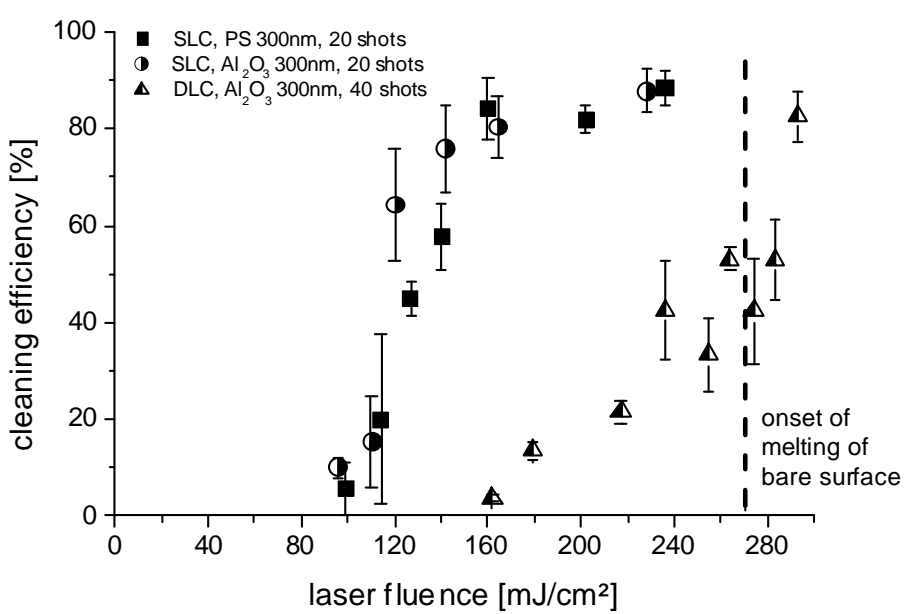

Results of these investigations are shown in Figs. 7 and 8. In the run presented in Fig. 7, 8 ns laser pulses $(\lambda=532 \mathrm{~nm})$ were used, as in the SLC measurements of Figs. 2 and 4. The data were obtained under ambient conditions in "normal" laboratory atmosphere at a relative humidity of $35 \%$. As the data demonstrate, cleaning is achieved also under these conditions. However, the efficiency is considerably reduced as compared to the steam cleaning data. Not only is the threshold for particle removal distinctly higher than for SLC, but even more importantly the slope of the efficiency curve is rather small, so that at the laser fluence of $270 \mathrm{~mJ} / \mathrm{cm}^{2}$, corresponding to the onset of melting of the Si surface under these conditions, 
only a cleaning efficiency of $50 \%$ is reached (in effect it is even worse, taking into account that not only 20 , but 40 cleaning pulses have been applied for each data point in this run).

Strictly speaking, the term "dry cleaning" is not really adequate to the experiment just described, because most surfaces under ambient conditions are covered with a water film whose thickness on a flat substrate is in the range of a few monolayers - depending on the humidity and chemical nature of the substrate - an in the gap between the substrate and the adhering particle might be considerably thicker due to capillarity. This film, although it is not as thick and well-defined as in the SLC, should due to the laser-induced evaporation assist particle detachment. It is to be expected, therefore, that the efficiency of "dry cleaning" should be further reduced if this film is removed by carrying out the experiment in vacuum.

Fig. 8: Cleaning efficiencies for Dry Laser Cleaning in ambient atmosphere and in high vacuum $(\mathrm{P}=10-6$ mbar $)$. The laser source was in this case an excimer laser (FWHM $30 \mathrm{~ns}, \lambda=248$ $\mathrm{nm})$. Due to the smaller optical penetration depth at this wavelength the cleaning threshold under ambient conditions is lower than for the DLC data in Fig. 7, where $\lambda=532 \mathrm{~nm}$.

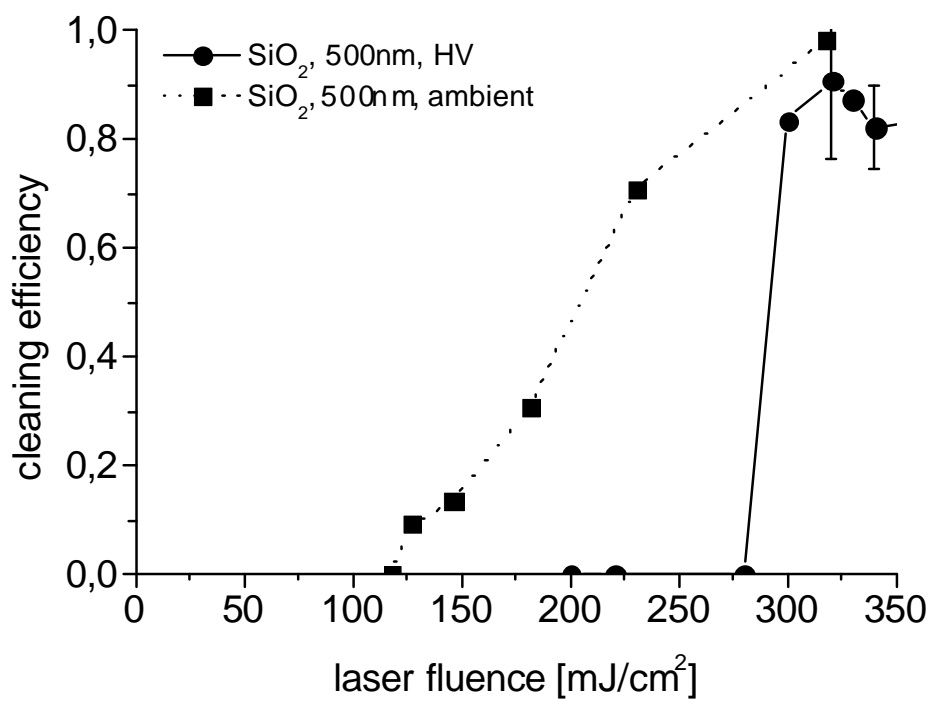

An example which confirms that this is really the case is presented in Fig. 8. Here a $\mathrm{KrF}$ excimer laser was used $(\lambda=248 \mathrm{~nm}$ and FWHM=30 ns), which is another common laser source for laser cleaning experiments. Two sets of data are shown, one of which was taken as DLC under ambient conditions, the other one in high vacuum (HV, pressure $\mathrm{p}=10^{-6} \mathrm{mbar}$ ). Although the water film is not completely removed in $\mathrm{HV}$, it is considerably thinner than at ambient pressure and usual humidity. Indeed, the effect of the HV condition is striking: the threshold is shifted to higher energies by more than a factor of 2 , which clearly shows the influence of the water layer on the particle removal process.

\subsection{Laser-induced surface damage}

We have already mentioned that the laser energy density below a small particle may exceed the homogeneous incident intensity by a large amount due to field enhancement. For transparent spherical particles with a diameter larger than the light wavelength this is obvious from geometric optics, because the sphere acts like a lens with a very short focal length. However, such a field enhancement is also relevant for smaller particles as shown below both by calculations and experiment. As the PS spheres used in our experiments are transparent for the wavelengthes of the cleaning lasers the field enhancement is of importance for the interpretation of the experimental data.

\subsubsection{Calculations of field intensities}

In Fig. 9 we present calculated field intensities near a dielectric sphere (PS, n=1.6) for three different particle sizes d, based on Mie calculations with a modified version of a program described in ref. 17. As a first approximation, we have neglected the influence of the silicon surface and performed the calculations for a particle in free space.

The field intensities are plotted both in a cross section perpendicular to the horizontal surface (a-c) as well as in the plane given by the substrate surface (d-f). Below the particle the energy density exhibits an enhancement by a factor of 30 for $\mathrm{d}=2 \lambda, 11$ for $\mathrm{d}=\lambda$ and about 2 for $\mathrm{d}=\lambda / 2$, and is localized into an area which is several hundred nanometers in diameter. 
These calculations corroborate that not only spheres with a diameter $\lambda>d$ focus light as expected from geometrical optics and demonstrated in ref. 18 , but also those with diameter $\lambda<\mathrm{d}$ enhance the field in their vicinity. The resulting patterns in the plane of the surface exhibit an anisotropy determined by the polarization of the incident light wave. Details of this field enhancement have been investigated systematically and will be presented in a separate publication [19].
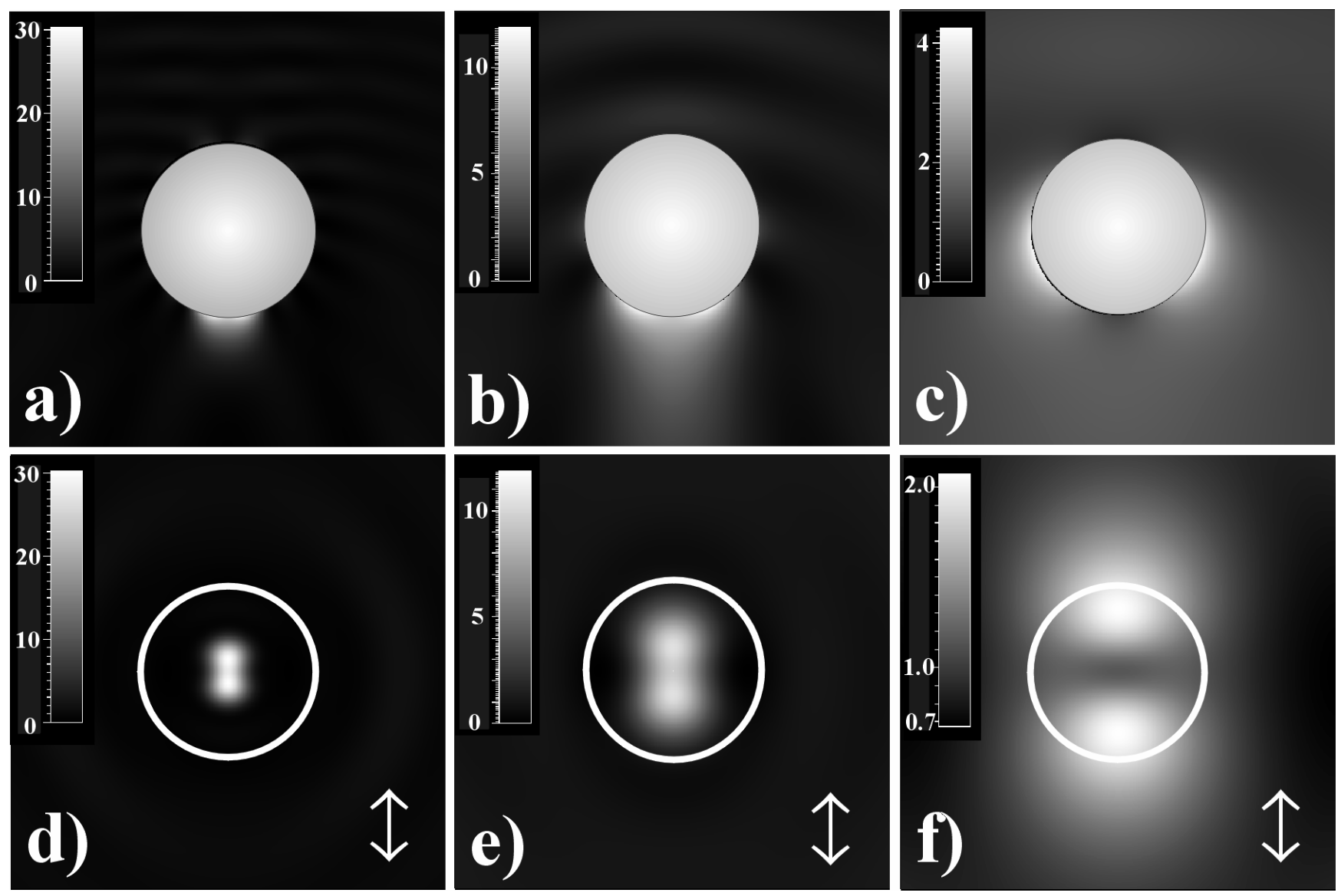

Fig. 9: Calculated field intensities for spheres (PS, refractive index $n=1.6)$ that are irradiated at $\lambda=800 \mathrm{~nm}$. The diameters of the spheres are $1700 \mathrm{~nm}(\mathrm{a}, \mathrm{d}), 800 \mathrm{~nm}(\mathrm{~b}, \mathrm{e})$, and $320 \mathrm{~nm}(\mathrm{c}, \mathrm{f})$. Plotted is the intensity in a cross section as seen from the side (a-c) and in the plane of the substrate surface as seen from above (d-f). The light field enters from the top, and the polarization vector of the electric field is indicated by an arrow. The displayed areas are 5100x5100 $\mathrm{nm}^{2}, 2400 \times 2400 \mathrm{~nm}^{2}$, and $960 \times 960 \mathrm{~nm}^{2}$, respectively.

These calculations reveal that in laser cleaning experiments one might deal with light intensities at the particle location which are considerably higher than the nominal energy densities incident on the wafer surface. This has consequences for the interpretation of all measurements of laser cleaning of transparent particles in which laser fluences are specified, especially the calibration of cleaning and damage thresholds. Even more seriously, the field enhancement below the particles leads to a high risk of modifying or damaging the surface.

\subsubsection{Experimental results}

We first discuss the effect of fs and ps pulses, where the spreading of thermal energy due to lateral and vertical heat diffusion in the substrate is negligible. Possible surface modifications are therefore much more influenced by the light energy distribution. 
The surface damage expected on the basis of field enhancement under small particles does indeed occur. When we examined wafers where colloid particles had been removed by fs or ps DLC we found holes all over the cleaned area. An example of a surface contaminated with $800 \mathrm{~nm}$ PS particles and cleaned with ps pulses $(\lambda=583 \mathrm{mn}, \mathrm{FWHM}=30 \mathrm{ps})$ is shown in Fig. 10. Three holes as well as a remaining colloidal particle can be seen. The hole shapes are elliptical with the long axis being parallel to the E vector of the incident light field, which is in agreement with the calculations mentioned above. AFM investigations of these holes gave a typical depth of $70 \mathrm{~nm}$ and a diameter of $250 \mathrm{~nm}$.

Fig. 10: A sample contaminated with $800 \mathrm{~nm}$ PS spheres after Dry Laser Cleaning with ps laser pulses. Elliptical holes can be found all over the illuminated region; a remaining particle can also be seen.

We have also marked the initial positions of the colloidal spheres by evaporating a thin (10 $\mathrm{nm}) \mathrm{SiO}_{2}$ layer onto the sample after application of the colloidal particles and before the laser cleaning step. After removal of the particles their size and position could be determined by a contrast in the SEM pictures due to the different thickness of the oxide layers in and outside the particle site. By a comparison of the hole diameters on wafers with and without the additional oxide layer we have checked that this layer did not influence the optical properties of the particles.
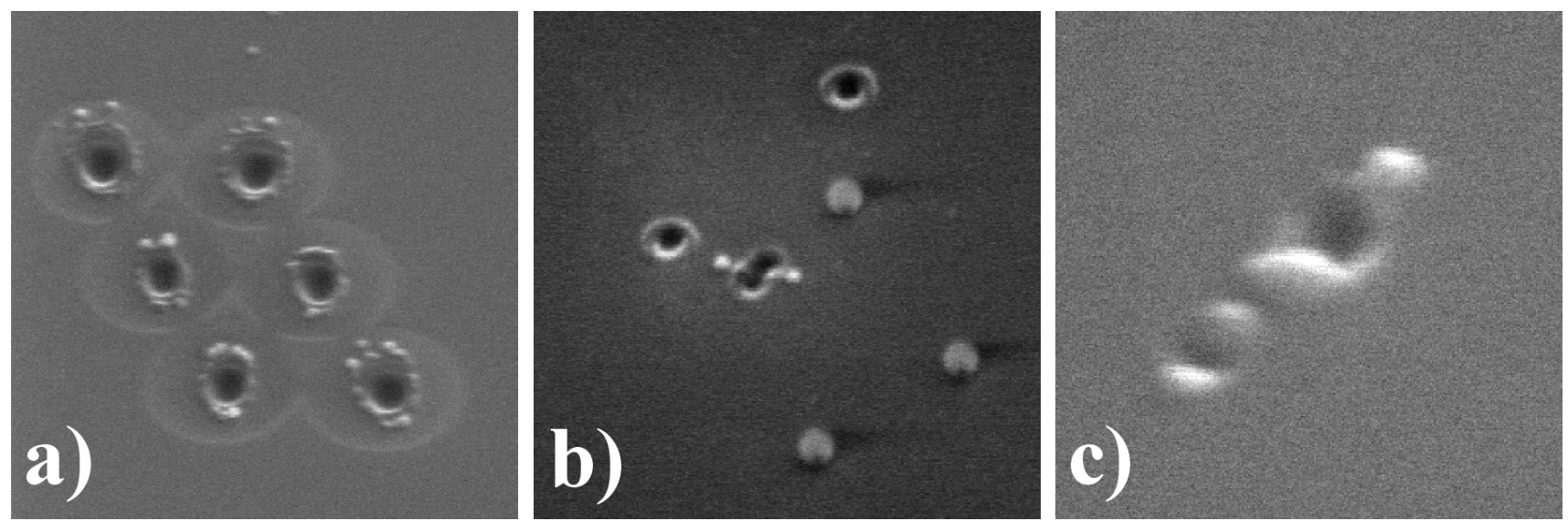

Fig. 11: Holes generated during DLC of silicon surfaces. In 11a and $11 \mathrm{~b}$ the particles were PS spheres with a diameter of 1700 and 320 $\mathrm{nm}$, respectively; in $11 \mathrm{c}$, irregular alumina particles with an average size of $400 \mathrm{~nm}$ were used. The side lengths of the displayed squares are $6800 \mathrm{~nm}, 4970 \mathrm{~nm}$, and $1300 \mathrm{~nm}$ for $11 \mathrm{a}, 11 \mathrm{~b}$, and $11 \mathrm{c}$, respectively. 
Fig. 11a shows the surface of a sample marked in such a way. The faint circular lines represent a shadowgraph of a cluster of six particles which were removed. At the center of each particle a hole was created, just at the positions where the numerical calculations predict the highest energy density. Similar holes were found also for the smaller particles (see Fig. 11b). The laser used here had a FWHM of $150 \mathrm{fs}$ and a wavelength of $\lambda=800 \mathrm{~nm}$. As it turned out in all the fs and ps DLC experiments, the removal of particles was always accompanied by the appearance of such holes. Hence the ablation process could in these cases be directly related to the ejection of material from the wafer, which then also leads to the observed crater formation.

Laser-induced holes are also observed on Si surfaces after DLC with short ns laser pulses. In this case lateral heat diffusion cannot be neglected, and therefore also the formation and shape of the holes that form at the same $\mathrm{d} / \boldsymbol{\lambda}$ - values is different. Compared to the illumination with fs and ps pulses, the hole size is larger and the shape becomes more circular, as it is qualitatively expected. On the other hand due to both lateral and vertical heat diffusion for longer ns pulses the defect size should decrease even more.

One might argue that realistic contaminants that have to be removed by laser cleaning applications are typically not of spherical shape. Also for irregular contaminants such as $\mathrm{Al}_{2} \mathrm{O}_{3}$ powder, however, field enhancement takes place - but in contrast to the spherical particles in asymmetric patterns - giving rise to the generation of holes also. An example of such a structure is shown in Fig. 11c.

In principle, field enhancement takes place not only in the DLC, but also the SLC process, and one might wonder why for SLC surface damage can much more easily be avoided. Three factors favor the SLC process in this respect:

- The cleaning efficiency at the same laser fluence is higher for SLC than for DLC, so one can work at lower intensity levels in the first place.

- For SLC, part of the absorbed energy flows into the water film, so that the silicon surface is heated only to a lower temperature at the same incident fluence.

- The water meniscus under the particle reduces the focussing effect of the dielectric particle and therefore the field enhancement.

\section{CONCLUSIONS}

The objective of the experiments presented here was to investigate the efficiency of particle removal by means of laser cleaning, and systematic studies of both Steam Laser Cleaning and Dry Laser Cleaning have been carried out. A broad spectrum of particle sizes, materials and shapes has been checked, and the laser pulse duration used for particle removal ranged from 100 fs to $29 \mathrm{~ns}$. The most promising results in terms of possible applications of laser cleaning for Si wafers have been obtained for SLC with ns and ps laser pulses. For the ns pulses a relatively broad window exists where efficient cleaning is achieved without damaging the silicon surface due to field enhancement effects. For ps SLC the laser fluence necessary for particle removal is lower, but the risk for crater formation at the surface due to field enhancement by the small particles may be higher, experimental investigations of this question are in progress.

For DLC, the cleaning efficiency of ns pulses is distinctly lower than for the SLC process, so that in order to reach technically interesting efficiency values well above $50 \%$ one has to apply a fluence which is already close to the melting threshold of the silicon surface, even without taking into account any field enhancement effects. DLC with shorter laser pulses always led to surface damage with crater formation under the conditions used here.

In summary, the combinations of different laser systems, particles and cleaning conditions studied in this work have yielded extensive sets of data, which are to be used for analyzing the fundamental mechanisms for laser-induced particle removal in more detail. Among the various scenarios, which are considered as being responsible for the particle removal process, two can already now be identified to dominate: i) for steam cleaning with ns pulses, explosive evaporation of the liquid film is the relevant mechanism; ii) dry cleaning with ps and fs pulses is mediated by the local ablation of the silicon substrate. A third scenario - DLC particle removal due to inertia forces, as mentioned in the introduction and so far considered to be the only mechanism responsible for this process - may play a role under certain conditions, e.g. pulses in the ns time regime, but 
normally either the presence of an „ambient“ water film or the local melting of the silicon surface will modify the detachment process. From the practical point of view, we conclude from our results that ns Steam Laser Cleaning is the most suitable laser cleaning method for a gentle detachment of even quite small particles from a wafer surface, and it appears to be applicable also on an industrial scale to complement more traditional cleaning methods.

\section{ACKNOWLEDGEMENTS}

This work was supported by the Optikzentrum Konstanz and the EU (TMR ERB-CT98-0188, "Modeling and diagnostic of pulsed laser-solid interactions: applications to laser cleaning"). The industrial silicon wafers were supplied by Wacker Siltronic.

\section{REFERENCES}

1. R. DeJule, “Trends in wafer cleaning”, Semiconductor International, August, pp. 64-68, 1998.

2. J. Bardina, "Methods for surface particle removal: a comparative study", Particles on surfaces, K.L. Mittal, pp. 329-337, Plenum, New York, 1988.

3. W. Zapka, W. Ziemlich, "Efficient pulsed laser removal of $0.2 \mu \mathrm{m}$ sized particles from a solid surface", Appl. Phys. Lett. 58, pp. 2217-2219, 1991.

4. K. Imen, J. Lee, S.D. Allen, „Laser-assisted micron scale particle removal“, Appl. Phys. Lett. 58, pp. 203-205, 1991.

5. A.C. Tam, W.P. Leung, W. Zapka, W. Ziemlich, „Laser-cleaning techniques for removal of surface particulates“, J. Appl. Phys. 71, pp. 3515-3523, 1992.

6. J.B. Heroux, S. Boughaba, 1. Ressejac, E. Sacher, M. Meunier, " $\mathrm{CO}_{2}$ laser-assisted removal of submicron particles from solid surfaces", J. Appl. Phys. 79, pp. 2857-2862, 1996.

7. Y.F. Lu, W.D. Song, K.D. Ye, M.H. Hong, D.M. Liu, D.S.H. Chan, T.S. Low, "Removal of submicron particles from nickel-phosphorus surfaces by pulsed laser irradiation", Appl. Surf. Sci. 120, pp. 317-322, 1997.

8. A.C. Engelsburg, "Transition from laboratory to manufacturing for a dry, laser-assisted cleaning technology", Proc. SPIE 3274, pp. 100-109, 1998.

9. G. Vereecke, E. Röhr, M.M. Heyns, "Laser-assisted removal of particles on silicon wafers", J. Appl. Phys. 85, pp. 3837-3843, 1999.

10. Interfacial Dynamics Corporation, Portland, Or 97224 USA

11. Duke Scientific Corp., 2463 Faber Place, Palo Alto, CA 94303, USA.

12. J. Siegel, J. Solis, C.N. Afonso, C. Garcia, "Bulk solidification and recalescence phenomena in amorphous Ge films upon picosecond pulsed laser irradiation”, J. Appl. Phys. 80, pp. 6677-6682, 1996.

13. M. Mosbacher, V. Dobler, J. Boneberg, P. Leiderer, „Universal threshold for the steam laser cleaning of submicron spherical particles from Silicon", accepted for publication in Appl. Phys. A.

14. P. Leiderer, J. Boneberg, M. Mosbacher, A. Schilling, 0. Yavas, „Laser Cleaning of Silicon Surfaces“, Proc. SPIE 3274, pp. 68-78, 1998.

15. M. Mosbacher, N. Chaoui, J. Siegel, V. Dobler, J. Solis, J. Boneberg, C.N. Afonso, P. Leiderer, „A comparison of ns and ps steam laser cleaning of Si surfaces“, Appl. Phys. A 69 [Supp.], pp. 331-334, 1999.

16. M. She, D. Kim, C.P. Grigoropulos, "Liquid-assisted pulsed laser cleaning using near-infrared and ultraviolet radiation", J. Appl. Phys. 86, pp. 6519-6524, 1999.

17. P.W. Barber, S.C. Hill, Light Scattering by Particles: Computational Methods, World Scientific Publishing, 1989.

18. S. Hayashi, Y. Kamamoto, T. Sutuki, T. Hirai: J. Colloid Interface Sci. 144, 538 (1991).

19. H.-J. Münzer, M. Mosbacher, J. Zimmermann, F. Burmeister, J. Boneberg, P. Leiderer: to be published. 